\title{
Segmentation and 3D Reconstruction of Cerebral Hematoma Based on Deep Learning
}

\author{
Taipeng Guo, Linbo Gong, Hongyu Deng, Lingrong Zhang, Yaohui Liu*, Tao Li \\ Xiangnan University, Chenzhou, Hunan \\ *Corresponding author.
}

\begin{abstract}
Cerebral hemorrhage is a common clinical disease. Because of its rapid onset, high mortality and disability rate, in the treatment of cerebral hemorrhage, it is very important to accurately calculate the brain hematoma volume and feedback its location information in a short period of time. This paper proposes a method for precise segmentation and three-dimensional reconstruction of cerebral hematoma area based on deep learning. This method highlights the image information by expanding the CT image and eliminating the skull information, then accurately segments the cerebral hematoma area through the neural network model to build a three-dimensional model. We verify the experimental results based on the data set collected by the Affiliated Hospital of Xiangnan University, which proves the effectiveness of this method and its ability to significantly improve the speed in cerebral hemorrhage area judgment and grasp information in clinical diagnosis.
\end{abstract}

Keywords: Cerebral hemorrhage, computer tomography (CT), computer-aided diagnosis, deep learning, three-dimensional reconstruction

\section{Introduction}

Among the numerous human brain diseases, cerebral hemorrhage ranks second in incidence, which is a common form of stroke. Although it has lower incidence than ischemic stroke, its fatality and disability rate are far higher than the latter [1]. China now has about 15 million stroke patients, and there are about 3 million new cases every year, so it is currently one of the countries with the heaviest stroke burden in the world. However, stroke treatment consumes abundant human and material resources, and there is a shortage of relevant professional doctors. Although there have been many programs for improving the stroke treatment and patients' prognosis, the treatment process costs a lot of resources and there are still high requirements for the professional skills of relevant physicians [2]. Therefore, timely and accurate determination of the patient's intracranial hemorrhage status (such as: hematoma shape, hematoma volume and location information) has always been the subject in many studies. The application of artificial intelligence technology, especially related knowledge in the field of deep learning and three-dimensional reconstruction methods, will effectively improve the detection accuracy and speed.

In the diagnosis of brain diseases, we need determine the hematoma shape, calculate the hematoma volume and acquire the location information of the hematoma in the skull, which is crucial to segmentation of the intracranial hematoma area in cerebral hemorrhage patients [3]. At present, radiologists' observation of medical images focuses on some special slices to identify some abnormalities in CT images. The accuracy of such diagnosis depends on the radiologist's experience [4]. In addition, the manual segmentation method requires a long segmentation time for a single image, and it is difficult to grasp the position information of the patient's hematoma in the actual scenario. For segmentation and detection of CT brain hemorrhage images, researchers have conducted many studies. In 2009, Mayank et al. published a paper on brain CT image [5]. The paper analyzes the image gray level to first remove the denser areas, and then analyzes the pixels of the brain CT image, retains the image information of a certain pixel value range, and then enhances the area of interest through opening operation. Moreover, two-level classification method is used to detect abnormalities to achieve the purpose of accurately segmenting the cerebral hematoma area.

ISSN: 0010-8189

(c) CONVERTER 2020

www.converter-magazine.info 
This method is only tested on a small patient data set, which may have certain limitations for large amounts of data. [6] In the paper, the cerebral hemorrhage area was segmented by CNN, but too many fully connected layers were used, resulting in excessive calculation load.

Therefore, this paper proposes a method for segmentation and three-dimensional reconstruction of cerebral hematoma based on deep learning. This method uses U-net [7] neural network to segment the cerebral hemorrhage area, and then uses VTK [8] to perform three-dimensional reconstruction of the result so that it is possible to achieve the purposes of accurately segmenting the cerebral hematoma area, determining the cerebral hematoma shape and acquiring the location information of the hematoma in the skull.

\section{The Method of This Paper}

The flow chart of the method herein is shown in Figure 1.

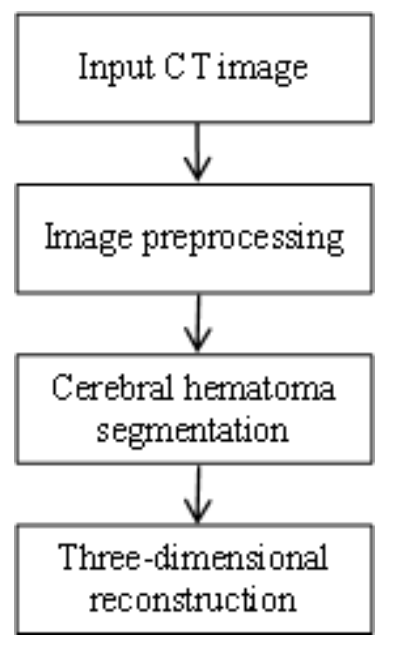

Fig 1: Method flow chart

\subsection{Image preprocessing}

First, we will preprocess the image. The NRRD image is in the form of $512 \times 512 \times n$. Where, $\mathrm{n}$ is the number of slices in the patient's brain CT image. Normally, the number of brain CT slices for a patient is between $20 \sim 30$. We convert the image from NRRD format to JPG format, then Gaussian filter the image through opencv and skimage[9] and use $3 \times 3$ cross-shaped convolution kernel for expansion, thereby eliminating the noise in the image and better retaining the image edge information.

When eliminating the skull information of CT images, we analyzed the distribution relationship of image pixel values, used threshold segmentation [10], ellipse fitting [11] and region growth algorithm [12] to extract the skull information. To better improve the image preprocessing speed and reduce the loss of effective information caused by this step, we choose threshold segmentation method to eliminate skull information. The result is shown in Figure 2 .

ISSN: 0010-8189

(c) CONVERTER 2020 

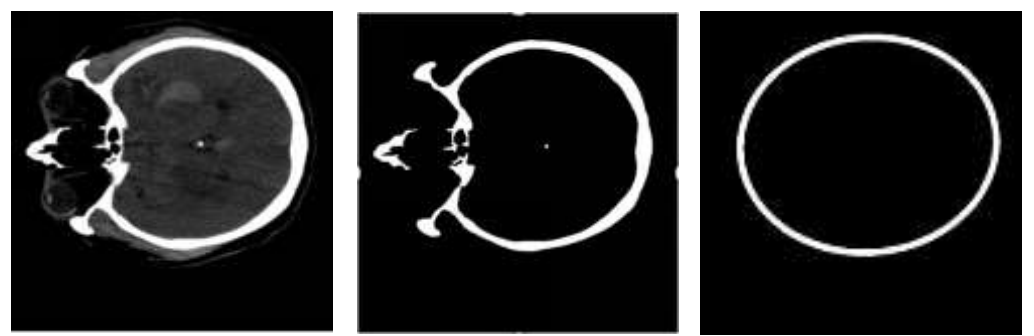

Volume 2021, No. 3

(a) CT image (b) Threshold segmentation result (c) Ellipse fitting result (d) Region growth result

Fig 2: Skull segmentation results

Only by image morphology changes, some areas irrelevant with the hematoma area may be generated, as shown in Figure 3.
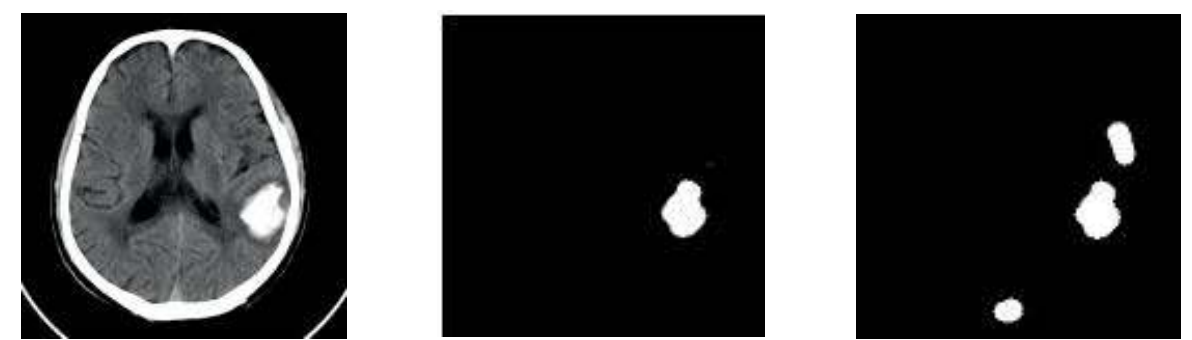

(a) CT image (b) Label image (c) Image with irrelevant area generated by morphological processing

Fig 3: Result of morphological error segmentation

As can be seen from Figure 3, mere morphological transformation will make some areas with similar pixel values to the hematoma confused, and incorrectly retain the corresponding results. Therefore, to better segment the cerebral hematoma area, we will further segment the image by training the neural network model.

\subsection{Segmentation of cerebral hemorrhage}

In our framework, the U-net network architecture is adopted, which is an architecture widely used in medical image segmentation. We implemented it through the pytorch framework.

The U-net network architecture is a FCN-based network structure in image segmentation [13]. Where, the fully connected layer in the traditional FCN is cancelled, and the ground size feature map is used instead. This method improves the network's utilization of spatial structure features. Application of U-net network structure can not only reduce the key information loss, but also integrate the feature map retaining massive information with the feature map whose key information is extracted. The U-net framework is shown in Figure 4.

ISSN: 0010-8189

(c) CONVERTER 2020

www.converter-magazine.info 


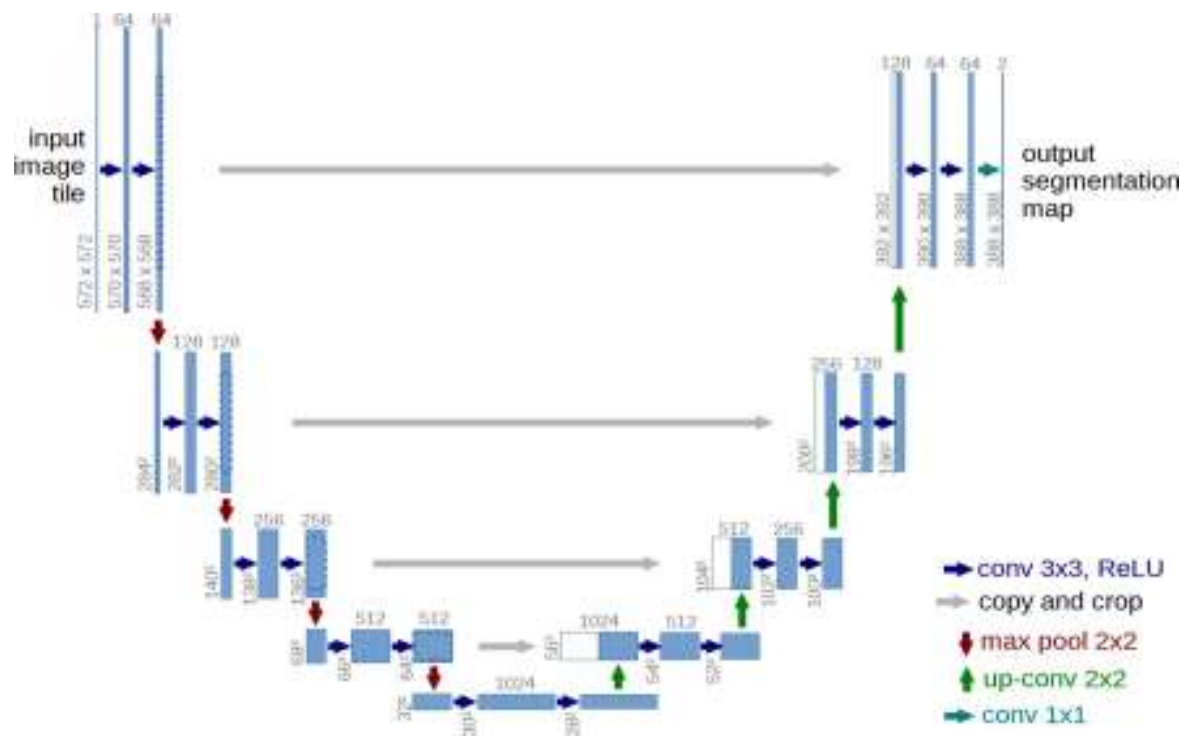

Fig 4: U-net framework diagram

The area of cerebral hemorrhage occupies a small area in a single CT image and displays few features. In response to these two problems, in our framework, the used gradient optimization algorithm is RMSProp adaptive gradient descent algorithm. This algorithm can eliminate the gradient descent swing in sparse data, so a higher learning rate can be used to increase the algorithm learning speed, as shown in Figure 5:

$$
\begin{gathered}
S_{d W}=\beta_{2} S_{d W}+\left(1-\beta_{2}\right) d W^{2} \\
W:=W-\alpha \frac{d W}{\sqrt{S_{d W}}+\epsilon} \\
S_{d b}=\beta_{2} S_{d b}+\left(1-\beta_{2}\right) d b^{2} \\
b:=b-\alpha \frac{d b}{\sqrt{S_{d b}}+\epsilon}
\end{gathered}
$$

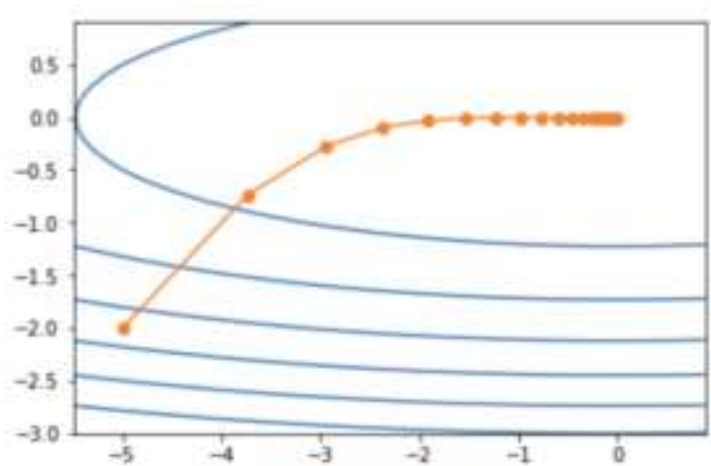

(a) RMSProp algorithm formula (b) Schematic diagram of gradient descent

Fig 5: Schematic diagram of the formula

In terms of loss function, we used a weighted cross-entropy function. Compared with the ordinary cross entropy function, this function is more suitable for images with imbalance between the target and the background, such as CT images. The formula of the weighted cross entropy function is as follows:

$$
\operatorname{WCE}(p, \hat{p})=-(\beta p \log (\hat{p})+(1-p) \log (1-\hat{p}))
$$

In the image preprocessing stage, we deleted the skull, bed position, etc. information in the image that greatly interferes with the network training. Therefore, in the network training stage, the hematoma segmentation will be more accurate to achieve a better segmentation effect.

2.3 Three-dimensional reconstruction

ISSN: 0010-8189

(c) CONVERTER 2020

www.converter-magazine.info 
Based on the accurate segmentation of the brain hemorrhage area, to better display the hematoma area location, we used VTK three-dimensional reconstruction library to perform three-dimensional reconstruction of the brain information.

We experimented and set an appropriate sampling point step size to enable more accurate image information data collection so that under the premise of ensuring the reconstruction speed, image quality is still a high. Secondly, we set the parameters of the reconstructed object properties, such as opacity, color, texture, light, etc. As different tissues in the brain have different CT values, we set different transparency and color for different CT value division thresholds to enhance spatial sense of the reconstructed model. Then, the problem of model edge sharpening due to sampling can be addressed by using vtkSetInterpolationTypeToLinear interpolation linear function, so that the reconstructed model is smoother and more aesthetic. Finally, the hematoma and the brain were displayed in the same window through Renderer and RenderWindow, and the vtkInteractorStyleTrackballCamera function was added to set the window interaction mode.
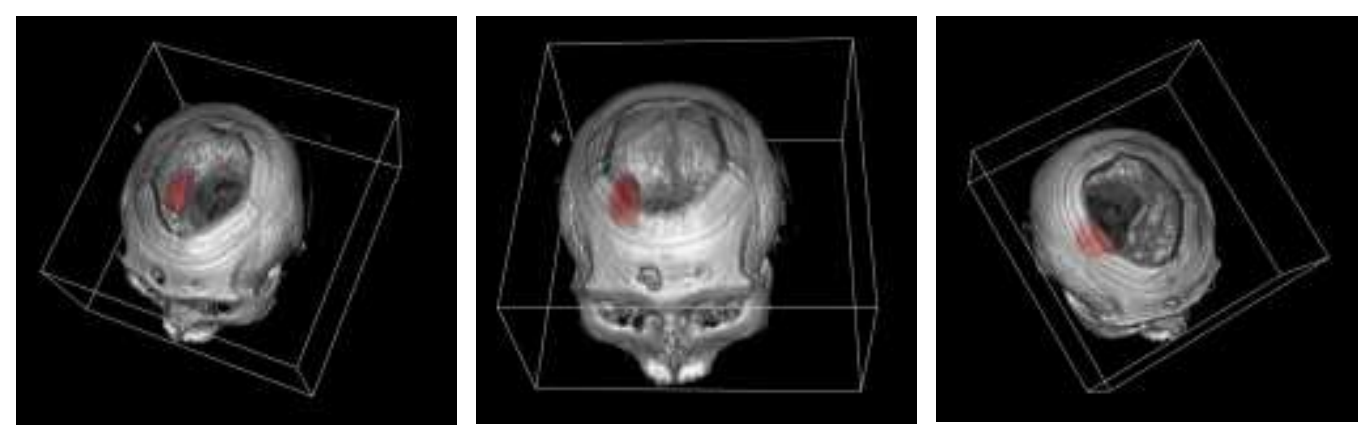

Fig 6: Three-dimensional reconstruction effect diagram

\section{Data set}

Our data set contains 412 sets of brain CT images, $20 \%$ of which were used for testing. All images were plotted by radiologists according to clinical standards, and the format is NRRD format. Its interior is composed of diffusion weighted image (DWI) volume and diffusion tensor image (DTI). NRRD is a way to transmit and store medical images, which can be recognized by a variety of medical devices.

When sampling, the data parameters were set as: layer thickness/layer distance: $5.0 \mathrm{~mm} \pm 0.3 \mathrm{~mm}$ field of view (FOV): 512x512 pixel resolution (Pixel Spacing): 0.48mm x 0.48mm.

CT image scanning is an image formed by mapping the amount of $\mathrm{x}$-ray absorbed by tissues in various parts of the human body to Hounsfield (HU) units through X-ray scanning. The amount of HU increases with the increase of tissue density. The various brain structures are complicated, such as skull, gray matter, white matter, hematoma, and edema [14]. By adjusting the window width and window level to filter out part of the CT image without brain information, the HU number was converted to gray $([0,255])$ values for deep neural network training.
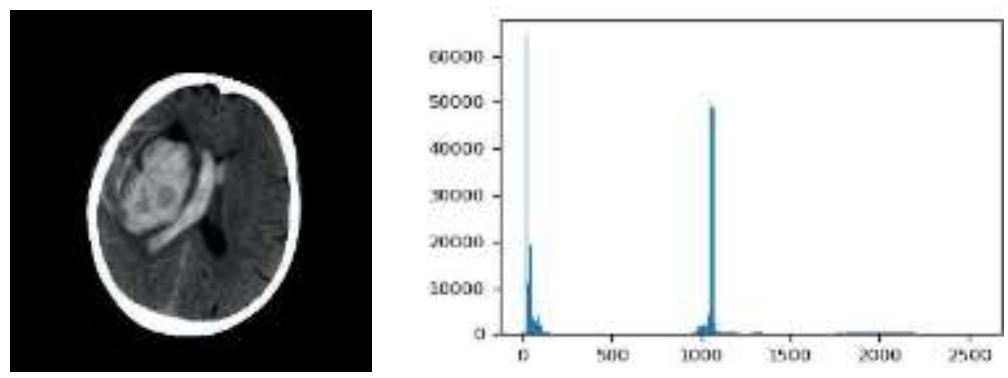

ISSN: 0010-8189

(c) CONVERTER 2020

www.converter-magazine.info 
Fig 7: CT image display and window width, window level distribution

The trained label data is manually annotated by the radiologist and provided in NRRD format. The impact of cerebral hemorrhage on individuals mainly depends on the compressed intracranial position and hemorrhage volume [15]. Hence, after training the model, we will perform three-dimensional reconstruction of the segmented cerebral hematoma to facilitate medical observation and treatment plan customization.

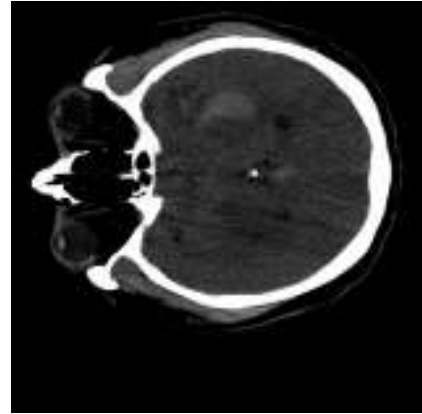

(a) CT image

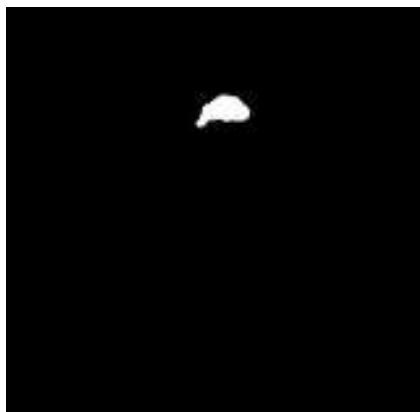

(b) label image

Figure 8 CT image and label

\section{Experimental Results and Analysis}

We processed the input image using convolution method. To ensure data consistency, when reading the CT image into the neural network, we first resized the image to a dimension of $512 \times 512$, thus reducing image information loss and avoiding potential integer division problems. We automatically adjusted the learning rate using RMSProp algorithm, and set the dropout_keep_prob parameter of the training model to 0.5. The model was trained for 300 epochs on our data set. The parameter table of the model is shown in Table 1.

Table 1 Parameter settings in U-net network

\begin{tabular}{|l|c|}
\hline \multicolumn{1}{|c|}{ Parameter } & Value \\
\hline Number of epoch & 300 \\
\hline Number of batches per epoch & 128 \\
\hline Size of image cube & $1 * 512 * 512$ \\
\hline Dropout keep probalility & $50 \%$ \\
\hline Kernel number for first layer & 64 \\
\hline Padding & SAME \\
\hline Kernel weights & $\mathrm{X}^{*} 3 * 3$ \\
\hline Number of convolutional layer & 9 \\
\hline
\end{tabular}
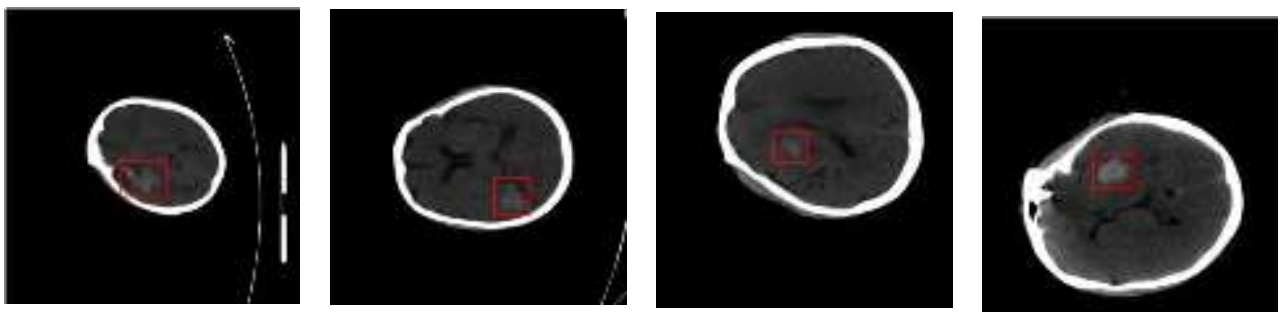

Fig 9: Cerebral hematoma segmentation results

The original CT image part, manual labeling and image segmentation results are shown in Figure 9. It can be seen that our network output achieves relatively good segmentation results, which can effectively reduce radiologists' workload and effectively speed up time-consuming tasks such as biopsy and small hematoma recognition. We can also calculate the hematoma volume through the skimage morphology image processing library to achieve better auxiliary diagnosis and treatment effect.

ISSN: 0010-8189

(C) CONVERTER 2020

www.converter-magazine.info 


\section{Conclusion}

Brain CT images are usually used by doctors to understand the internal conditions of the patient's brain and formulate treatment plans. This paper describes a method of using deep learning to learn the data manually annotated by imaging doctors so that hematoma segmentation for the brain MRI image is possible. Based on this, three-dimensional brain information reconstruction is performed. This method is robust to the segmentation and detection of medical images, and its output can enable doctors to better judge the patient's condition. Our method has a good segmentation effect on cerebral hemorrhage, but it is temporarily unable to distinguish the types of cerebral hemorrhage. As a result, our future research direction is to accurately judge the cerebral hemorrhage type, thus better and more accurately assisting the doctor's diagnosis and treatment.

\section{References}

[1] X.Y. Hong, P.N. Liu, B.F. Zhu, "Research progress in factors related to lumbar intervertebral disc degeneration," Chinese Manipulation \& Qi Gong Therapy, vol. 11, no. 10, 2020.

[2] G.Q. Zang, D. Ye, "Diagnosis and Clinical Value of CT for Cerebral Hemorrhage," Chinese Journal of CT and MRI, vol 14, no. 4, April, 2016.

[3] J.D. Lai, N. Wang, K. Luo, "Measurement of cerebral hemorrhage volume based on deep learning computer-aided diagnosis system," Chinese Journal of Medical Imaging Technology, vol. 36, no.12, 2020.

[4] S. Ray, V. Kumar, C. Ahuja, "Intensity population based unsupervised hemorrhage segmentation from brain CT images,” Expert Systems With Applications 97, pp 325-335, 2018.

[5] Y. Qin, G.L. Li, X.H. Xu, et al. "Brain structure alterations and cognitive impairment following repetitive mild head impact : An in vivo MRI and behavioral study in rat," Behav Brain Res, vol. 340, pp. 41-48, 2018.

[6] C. Chin, G. Wu, C. Yang, "An automated early ischemic stroke detection system using CNN deep learning algorithm. 8th International Conference on Awareness Science and Technology (iCAST), Nov $1,2017$.

[7] O. Ronneberger, P. Fischer, T. Brox, U-Net: Convolutional Networks for Biomedical Image Segmentation, arXiv:1505.04597, 5.18, 2015.

[8] M.M. Wang, "Design and Implementation of 3D Reconstruction System for Vertebral CT Image Based on Improved Ray Casting," Harbin University of Science and Technology, pp. 4, 2020. http://scikitimage.org/docs/dev/api/skimage.measure.html\#skimage.measure.regionprops

[10] L. J. Kong, Q. W. Wang, Y. C. Bao, “Overview of Medical Image Segmentation Based on Deep Learning," Radio Communications Technology, pp. 2, 2012.

[11] N. Wang, H.H. Song, K.H. Zhang, "Accurate target tracking algorithm based on distance weighted overlap estimation and ellipse fitting optimization, Journal of Computer Applications," pp. 1, 2021.

[12] S.Y. He, "Salient target segmentation algorithm based on region growth," Harbin Institute of Technology, pp. 6, 2019.

[13] X.J. Yin, Y.C. Wang, D.Y. Li, “Overview of Medical Image Segmentation Technology Based on U-Net Structure Improvement," Journal of Software, pp. 2, 2021.

[14] W. J. Jia, S.H. Wang, Y.Z. Zhang, "Review of imaging-based diseased brain detection methods," Computer Engineering and Applications, vol. 54, no. 11, 2018.

[15] J.B. Chang, T.J. Jiang, X.J. Chen, “Consistency evaluation of spontaneous intracerebral hemorrhage hematoma segmentation method based on convolutional neural network," Chinese Journal of Contemporary Neurology and Neurosurgery, July 2020, vol. 20, no.7, 2020.

ISSN: 0010-8189 